\title{
Health procrastination: The experience of 35-44 years old men
}

\author{
V. Silkane ${ }^{*}$ and I. Austers \\ University of Latvia, Riga, Latvia
}

\begin{abstract}
The focal concept of this study is health procrastination. Delay in seeking help from medical practitioners is an increasing problem in Latvia, particularly with men between the ages of 35-44. Failures in primary and secondary prevention create a number of negative consequences, for instance, longer and more complex treatment; lower recovery prognosis as well as higher treatment costs. Nevertheless, the aforementioned group often avoids medical treatment. The aim of this study was to understand the experience of 35-44 years old men in terms of their health procrastination in qualitative terms. Semi-structured in-depth interviews with 48 men within the age range of 35-44 years were conducted. Data was analysed using inductive thematic analysis. The following dominant themes are: delay of health behaviour, time management, motivation emerged during the analysis: conscientiousness, task avoidance, effort, short-term vs. long-term thinking, gains and losses assessment, and time discounting. Conclusions followed: there are three main concepts - personality traits, discounting and task characteristics that bring together the experience of health procrastination for 35-44 years old males.
\end{abstract}

\section{Introduction}

Failure to take care of one's health in a timely manner is a wide-spread problem that is often debated, but at the same time has shown itself lacking a clear solution. Taking care of own health means both leading a healthy life-style and seeking medical assistance in a timely manner. Very often, unless health problems are severe, like acute pain or other distressing symptoms, people simply neglect them. For example, a 2014 survey on health related habits of inhabitants of Latvia, carried out by The Centre for Disease Prevention and Control [1], showed that $68.9 \%$ of respondents had never visited a general practitioner to have a free medical check-up (75.3\% men and $62.7 \%$ women) and $27 \%$ of respondents had not visited a general practitioner during the past year (35.9\% men and $18.4 \%$ women). Those who most commonly do not visit general practitioners are men aged 25-34 (43.4\%) [1] and men aged 35-44 (47.8\%) [2]. A delayed or a postponed visit to a doctor, a failure to abide the treatment instructions and to take preventive measures cause several problems illnesses that require a longer and more complicated treatment, worse recovery forecasts, as well as higher treatment costs.

\footnotetext{
${ }^{*}$ Corresponding author: vineta.silkane@gmail.com
} 
Even though in the long term people regard health as an important asset, they often postpone actions in this respect - they not only miss preventive check-ups and visits to a doctor, they also do not follow practices related to a healthy life-style. Despite possessing knowledge associated to a healthy life-style, people postpone the cessation of smoking, starting physical activities, and following healthy nutrition habits [1].

Therefore it is necessary to examine factors related to postponing health behaviour and delay in taking care of one's health, in other words - health procrastination.

An important group that deserves additional research attention is men aged 35-44. At this age men often get their first health problems in addition to the unhealthy life-style they have, for example, smoking, eating unhealthy food, and suffering from overwork and stress. At the same time men in this age group rarely visit doctors to solve health-related issues [2]. Thus, men falling within this age group have a bigger risk of not noticing health problems. In order to gain a better understanding of men's health procrastination and the most common reasons why men neglect their health, it is important to apply the methods of a qualitative approach.

Men's health behaviour is studied fairly rarely and is mostly formed by quantitative research design dealing with masculinity and sexuality topics $[3,4]$. In order to conduct a better examination of men's health behaviour experience and the most common reasons of not caring for their health, it is important to use a qualitative study approach. The aim of the study was to explore the experience of 35-44 years old men in terms of their health procrastination.

\subsection{Procrastination}

In the absence of a unified definition [5] the majority of researchers focus on three criteria to define procrastination: (1) activity is delayed or postponed, (2) postponement of activity is unnecessary and (3) activity is unproductive [6-8]. Since it is difficult to formulate nonnecessity and non-productivity in an objective way, definitions focus more on delayed or postponed activity [5].

Procrastination is the voluntary [9] and irrational delay of intended activities or postponement of tasks [10-13], despite the expected aggravation of the situation [9]. Irrationality is characterised by the fact that such behaviour is chosen even though it is unsuitable and contrasts the individual's material (e.g., financial) or psychological (e.g., wellbeing) interests or objectives. An individual opts for worsening their situation although other solutions exist [15]. If hesitation is rational or has a sensible explanation, it is not procrastination [12].

Klingsieck [5], after analysis of the most popular definitions, clearly distinguishes between procrastination and functional forms of delay, like strategic delay. Taking into account everything written above, procrastination is "the voluntary delay of an intended and necessary and/or [personally] important activity, despite expecting potential negative consequences that outweigh the positive consequences of the delay" $[5,9]$.

The most common explanations as to why procrastination emerges are (1) time discounting, (2) task characteristics and (3) personality traits $[9,14,15]$. The majority of studies focus on procrastination as a personality trait. Some researchers suggest that procrastination can be reduced by changing behaviour $[11,16]$. Task characteristics are analysed less profoundly, for example, when an individual perceives the task as insignificant, difficult, aversive or boring $[17,18]$ tasks with failure potential [19-21] and whether there is a deadline for the task $[21,22]$. 


\subsection{Procrastination and personality}

In numerous studies procrastination is conceptualized as a personality trait [10]. Procrastination can be examined as a personality trait that is created by repeated procrastination behaviour [7]. If procrastination is constant over time and stable in various situations [9], procrastination is regarded as a personality trait.

Procrastination is related to a low conscientiousness level $[9,19,23-25]$ as well as to a high neuroticism level [9, 19, 23-25]. For self-disciplined, accurate individuals it is much easier to meet deadlines and overcome resistance against aversive and tough tasks, therefore it is less likely that they would procrastinate. Impulsivity explains how neuroticism is related to procrastination. For instance, individuals would more often postpone fulfilment of tasks if there is strong drive for a more pleasant activity, and their self-control is too weak to be able to overcome desires, put forward by the moment, and to do what is necessary instead. For impulsive individuals it is difficult to get over their desires and they tend to succumb to various temptations and enticements [7]. Due to the lack of impulse control they are unable to postpone immediate satisfaction; at the same time they are unable to refuse pleasant short-term benefits for the sake of long-term results [19]. Studies that have used the Big Five personality model do not render significant results regarding the relationship between procrastination and extraversion, openness and agreeableness.

\subsection{Procrastination and discounting}

Some researchers argue that procrastinators avoid focusing on future tasks and that procrastination is related to time perception [26]. In studies that examine procrastination from the perspective of time, procrastination is most commonly described using time discounting [24, 27, 28]. Procrastinators frequently do not appreciate the importance of present efforts in relation to future achievements [24]. Even though the future benefit is very much welcome, or drawbacks are dangerous and terrible, it is difficult for an individual to get over discounting [28]. The longer one has to wait to get a reward for the particular behaviour, the less attractive that behaviour seems [24]. Future results are discounted or undervalued in comparison with immediate benefits [14].

Discounting relates to higher procrastination levels [27]. From the point of view of discounting, it is considered that procrastination develops when present costs seem higher than the future costs. Discounting of future costs may, at least partially, be explained by topicality of other activities that are to be done in the near future; people mostly believe that they will have more time to do it in the future [29] therefore procrastination often may be limited by setting deadlines or defining a limited time-frame [30].

\subsection{Procrastination and task characteristics}

Several studies have defined that one of the main causes of procrastination is averse, boring or uninteresting tasks $[9,15]$. Procrastination studies usually define task avoidance by asking study participants to grade it [31] and it usually means boredom, frustration and offence. Task avoidance has a positive correlation with procrastination [18, 32].

People procrastinate more often if they have to do unpleasant tasks or the task is expected to be unpleasant $[9,14]$, it requires more effort, it is vaguely defined, less pleasing [26], tough and unattractive $[18,33,34]$. 


\subsection{Procrastination and health behaviour}

Relatively few studies have explored the relationship between procrastination and health behaviour. There are studies that examine the role of procrastination in the explanation of illness and each of them uses and analyses different mediators: (1) stress as a mediator between procrastination and illness $[35,36]$ and (2) treatment delay as a mediator between procrastination and illness $[35,36]$. One of the studies has addressed the relationship between procrastination and health behaviour intention [37] and the mediators are selfefficiency and future consequences [37].

Procrastination is related to a higher stress level and worse health [38], anxiety and depression [31]. Procrastination is also related to more acute health problems, nonpracticing of a healthy life-style, rarer visits to the doctor [36], and postponement of looking for help and treatment [36].

\section{Method}

Participants. Participants were recruited by using snowball sampling and participation in this study was voluntary. Forty-eight individual interviews were conducted among 35-44 year old men, the average age of the sample was $M=40.44, S D=3.96$. When quoted, study participants were identified by code number and age (for example, A15, 37).

Data collection. Semi-structured in-depth interviews were used as a data collection method. Interview questions were formulated in the way that would be possible to find out the instances of participants' health procrastination, as well as examining the following topics: taking care of health, healthy lifestyle motivators, reason for not taking care of health, delay or postponement of health behaviour or health checks. Participants were informed about the confidentiality of any information collected.

Data analysis. Inductive thematic analysis was used. First, repeated examination of each interview took place. Then, the search and definition of topics and sub-topics was performed.

\section{Results}

The aim of the study was to explore the experience of 35-44 years old men in terms of their health procrastination. The interviews allowed for the identification of the following behaviours related to procrastination: postponement, laziness, effort, lack of motivation, impulsivity, time management, task avoidance, assessment of gains and losses, discounting.

Several men mentioned health behaviour postponement. It was shown to manifest through postponement of a visit to a doctor because one "has to wait for a better blood test if too many sweets have been consumed" (the man has diabetes mellitus) (A33, 36). Or it may be a prevention postponement: "generally, the idea is good, but sometimes later (A38, 39). Another participant characterised men's behaviour: "Men tend to postpone solving problems as much as possible and finally get used to them. The reaction follows only after a problem has occurred" (A47, 35). It can be explained by short-term planning, assuming that the symptoms will vanish and no actions will be necessary. However, such an approach can be a problem solving model. An example mentioned by another man illustrates it: "One has to reach an edge of a catastrophe and then it happens" (A42, 44). One of the men postponed wisdom tooth extraction because the previous time he had done it, recovery took him 3-4 days and he could not find time for that. He postponed it "until an emergency situation occurred, there was sharp pain and nothing could be done about it anymore" (A48, 37). These examples show that postponement can make the situation worse.

Sometimes men evaluate the situation in terms of pros and cons, which can be regarded as a strategic postponement mentioned by Klingsieck [5]. For example, the postponement 
of an operation, since it affects working capacity in the long-term. "I have no spare six months in which I can afford to do nothing. Therefore I wait and hope that maybe some new technologies will be introduced. It does not affect my daily life; the only thing is that I can't play basketball" (A40, 43). Another man acted in a similar way, he postponed removal of a metallic element from his arm after a fracture because he had difficulties in finding a time for an operation. In both situations, postponement of surgery did not affect the daily routine and had no harmful consequences therefore one cannot speak of health procrastination in these particular situations.

Men often explain not doing something, for example, postponement of physical activities, by laziness. For example, "I am too lazy for running" (A3, 37). At the same time the man added that "as to health matters, I am not lazy," because "I value health very much" (A5, 43). When explaining the phenomenon of laziness, lack of motivation is often mentioned, as well as the effort that needs to be present to achieve something: "One has to have motivation in order to start doing things" (A38, 39). In this case attention has to be paid to the use of terms, as 'laziness' in its daily use does not always mean the same as the scientific definition of the term. Steel [39] is of an opinion that laziness and procrastination are contrasting concepts that only partly overlap, but for the purpose of this study it was important to take into account all kinds of explanations that are related to procrastination. A failure to do something or a postponement of doing things is something that laziness and procrastination have in common. The same applies to a lack of motivation. Both concepts differ in terms of feeling bad and guilty, which is present in case of procrastination, while it is not characteristic of laziness [39].

In order to follow different tasks, including engagement in health related activities, effort is required. It is known that procrastination is more widespread if the task seems aversive, difficult or boring. When explaining their behaviour, men often discussed activities that require effort, meanwhile acknowledging that they lack the willpower for that. Cessation of smoking and taking part in physical activities are among activities mentioned most often when speaking about effort, for example, "I have tried [to quit smoking], but I do not have enough willpower" $(\mathrm{A} 2,44)$, or "I am tired after work therefore it is difficult to do sports" (A31, 42) and "I would like to [engage in physical activities], but I lack determination" (A36, 44). In the example about wisdom tooth extraction given in the discussion previously, another source of procrastination related to the task characteristics appears - task avoidance.

In the interviews the men talk about lack of motivation, as well as mention that finding the right motivation is a key for success. For example, one man said that there are "a thousand reasons not to" (do sports), but "I decided to lose some waist weight" (A33, 36) and it helped to start. Another man explained that he will not choose a healthy life-style because he does not believe in it and the results do not double life expectancy anyway: "If I was sure that quitting smoking or eating carrots would allow me to live up to 150 years, maybe I would do that" (A14, 44). Such approach illustrates lack of motivation.

One of the interviews revealed impulsivity, which is a common companion of procrastination: "I instantly decide to do something and if it is not possible straight away most likely my instant decision will be gone" $(\mathrm{A} 42,44)$. In this particular case the man speaks about paying a visit to a doctor and about a preventive examination.

Frequently in their explanations men talk about time management. Lack of time may be connected with difficulties in time-planning "[...] time, lack of time has always been an obstacle" (A4, 38), as well as scrupulosity. One man stated that time management is "a matter of self-discipline" and "it is hard to be regular enough to make particular activity a tradition" (A48, 37).

Assessment of gains and losses was also present in the interviews and it may characterise short-term vs long-term thinking. For example, "If a dentist discovers cavities 
in teeth, it is cheaper and less painful. [...] If I don't visit a dentist, at the end the bad tooth has to be extracted and it costs thousands to replace it, or else I have to go on without a tooth" (A44, 35). The example about postponement of a surgery mentioned before portrays a similar situation. The man has already considered that maybe the technologies will improve in the future while shoulder trauma is bearable in his daily life. Sometimes such choice can be explained by discounting, since an immediate but smaller benefit is chosen. For example, "Men tend to postpone the solving of problems as much as possible and finally get used to them. Reaction follows only after problem occurrence" (A47, 35) or "I postpone going for a jog because I choose to watch a movie instead" (A44, 35). It can be seen also in the quotation given above where the man says that a healthy life-style will not extend his life expectancy up to 150 years, therefore it is not even worth starting because "[...] a man has as a pre-destined length of life either way" $(\mathrm{A} 14,44)$ and, at its best, it can be prolonged for one or two years.

\section{Discussion and conclusions}

The thematic analysis of interviews proves that stories told by the men allow for the identification of health procrastination and related concepts, thus, characterising the circumstances of procrastination occurrence. The categories mentioned in the results section can be divided into three groups which comply with the three most common procrastination explanations mentioned in the research literature - task characteristics (e.g., effort, unpleasantness), personal traits (e.g., impulsivity, conscientiousness) and discounting. However, further quantitatively designed studies are needed, in order to verify the role of all three concepts used in the explaining of procrastination as a single model.

The limitation of the study is gender difference between the research participants and the interviewer; it may have an impact on what and how answers are given about health behaviour and health procrastination. It is probable that other health-related nuances would have been revealed and slightly different behaviour explanations would have been given to a male interviewer.

The results of the study can be useful for the development of a relevant health policy. Currently, health policy in Latvia is using a variety of interventions, for example, informing individuals about healthy lifestyle and health-related regulations. However, the psychological aspects are not always taken into account, for example, when trying to understand an individual's decisions and the possibilities to influence those decisions. For example, in the case of restricting smoking both information and various regulations are used; yet, despite the fact that people know about the dangers of smoking, quitting is delayed. More severe regulations and restrictions do not necessarily have positive results, since the forced behaviour rather creates resistance than confirms the expected patterns. Therefore, there is a need for developing interventions that promote a healthy lifestyle and at the same time are based on understanding the psychological factors affecting a healthy lifestyle.

At the level of public health, it is difficult to create a health intervention that could change all aspects contributing to health procrastination, like personality traits, that the study illuminates. However, it is likely to influence the intentions related to a healthy lifestyle, as well as preferences, and motivation. It is also possible to reduce the systemic barriers related to health behaviour and thereby reduce effort to implement a healthy lifestyle, for example, to make preventive visits to the doctor more routine. Special attention should be paid to men's health-related campaigns. They should focus on the evaluation of benefits, losses, and risks in the long run. 


\section{References}

1. The Centre for Disease Prevention and Control, Health Behaviour among Latvian Adult Population (2014)

2. The Centre for Disease Prevention and Control, Health Behaviour among Latvian Adult Population (2012)

3. J.R. Mahalik, M. Levi-Minzi, G. Walker, (2007). Psychol. Men Masculin. 8, 240249 (2007)

4. T. Calasanti, I. Pietilä, H. Ojala, N. King, N., Health Psychol, 32, 15-23 (2013)

5. K.B. Klingsieck, Eur. Psychol. 18, 24-34 (2013)

6. H.C. Schouwenburg, Procrastination and Task Avoidance: Theory, Research, and Treatment, 71-96 (1995)

7. H.C. Schouwenburg, C.H. Lay, Pers. Individ. Dif. 18, 481-490 (1995)

8. G. Schraw, T. Wadkins, L. Olafson, J. Educ. Psychol. 99, 12-25 (2007)

9. P. Steel, Psychol. Bull. 133, 65-94 (2007)

10. C.H. Lay, J. Res. Pers. 20, 474-495 (1986)

11. J.B., Burka, L.M. Yuen, Procrastination: Why You Do It, What to Do About It (2004)

12. M. Silver, J. Sabini, J Theory Soc Behav, 11, 207-221 (1981)

13. C. Andreou, J. Theory Soc. Behav. 37, 183-193 (2007)

14. J. Wistrich, Wm. \& Mary L. Rev. 50, 607-667 (2008)

15. Wilson, T.D. Nguyen, Int. J. Psychol. Res. (Medellin) 4, 211-217 (2012)

16. W.J. Knaus, J. Pers. Soc. Psychol. 15, 153-166 (2000)

17. D.S. Ackerman, B.L. Gross, J. Mark. Educ. 29, 97-110 (2007)

18. K. Blunt, T.A. Pychyl, Pers. Individ. Dif. 28, 153-167 (2000)

19. W. Van Eerde, Pers. Individ. Dif. 35, 1401-1418 (2003)

20. C.H. Lay, J. Pers. Soc. Psychol. 5, 91-103 (1990)

21. R. Gupta, D.A. Hershey, J. Gaur, Curr. Psychol. 31, 195-211 (2012)

22. J.R. Ferrari, Pers. Individ. Dif. 13, 315-319 (1992)

23. D.C. Watson, Pers. Individ. Dif. 30, 149-158 (2001)

24. S. Dewitte, H.C. Schouwenburg, Eur. J. Pers. 489, 469-489 (2002)

25. P. Steel, T. Brohen, V. Wambach, Pers. Individ. Dif., 30, 95-106 (2001)

26. J.R. Ferrari, C.P. Mason, C. Hammer, Individ Differ Res 4, 28-37 (2006)

27. J. Howell, D.C. Watson, R.A. Powell, K. Buro, Pers. Individ. Dif. 40, 1519-1530 (2006)

28. H.C. Schouwenburg, J. Groenewoud, Pers. Individ. Dif. 30, 229-240 (2001)

29. S.B. Shu, A. Gneezy, J. Mark. Res. 47, 933-944 (2010)

30. D. Ariely, K. Wertenbroch, Psychol. Sci. 13, 219-224 (2002)

31. L.J. Solomon, E.D. Rothblum, J. Couns. Psychol. 31, 503-509 (1984)

32. K. Krause, A.M. Freund, Eur. Psychol. 1, 1-13 (2013)

33. D.S. Ackerman, B.L. Gross, J. Mark. Educ. 27, 5-13 (2005)

34. C.H. Lay, J. Pers. Soc. Psychol. 7, 483-494 (1992)

35. F. M Sirois, M.L. Melia-Gordon, T.A. Pychyl, Pers. Individ. Dif. 35, 1167-1184 (2003)

36. F.M. Sirois, Pers. Individ. Dif. 43, 15-26 (2007)

37. F.M. Sirois, Pers. Individ. Dif. 37, 115-128 (2004)

38. D.M. Tice, R.F. Baumeister, Psychol. Sci. 8, 454-458 (1997)

39. P. Steel, The Difference Between a Procrastinator and a Lazy Person [Video file], 2016 
